\title{
X-ray Fluoroscopy-Guided Method
}

\author{
Hiroki lida
}

\subsection{Introduction}

Image-guided intervention techniques are used for performing procedures with safety and accuracy. Such procedures for pain treatment can be performed for both maximizing benefit and minimizing the risks to the patient. In general, fluoroscopy using $\mathrm{C}$-arm devices and ultrasound are the most commonly used forms of image-guided intervention in pain treatment. However, fluoroscopy-guided method can only recognize bones; thus, they are used only for target nerves expected in the vicinity of a bony landmark.

\subsubsection{Advantages}

1. The position of the needle with respect to the bony landmark can be visualized with a wide field of view in the target region allowing practitioners to correctly place needles for accurate delivery.

2. Intravascular injection can be relatively easily detected (compared to ultrasound-based techniques) using a contrast medium, and this increases the clinical efficacy, decreases possible side effects, and enhances patient safety.

\subsubsection{Disadvantages [1]}

1. There is the potential for adverse effects to patients and staffs, who undergo prolonged exposure to fluoroscopy. To minimize radiation exposure, the following steps can be taken: (1) intermittent use of fluoroscopy; (2) placing the image intensifier as close to the patient as possible; (3) the target region image should be kept in the center of the field, and the size of the x-ray field should be reduced as much as possible; and (4) the use of suitable shields (such as a thyroid shield and leaded gloves) could help decrease exposure to the staff.

2. Since it is necessary to imagine the three-dimensional anatomy while watching the two-dimensional X-ray images, practitioners are required to learn and develop their skill.

3. The possibility of an allergic reaction to the contrast medium commonly used during fluoroscopic procedures.

Recently some pain clinicians have started using a CT-guided method instead of fluoroscopy, which allows for a safer placement of the needle.

\section{Reference}

1. Wang LH, McKenzie-Brown AM, Hord AH. Radiation safety. Handbook of fluoroscopy-guided spinal injections. Boca Raton, FL: Taylor \& Francis; 2006. p. 29-40.

H. Iida $(\bowtie)$

Department of Anesthesiology and Pain Medicine,

Gifu University Graduate School of Medicine,

Gifu, Japan

e-mail: iida@gifu-u.ac.jp 